\title{
Bullet Time Using Multi-Viewpoint Robotic Camera System
}

\author{
Kensuke Ikeya \\ $\mathrm{NHK}$ \\ 1-10-11 Kinuta Setagaya-ku \\ Tokyo, Japan \\ ikeya.k-ec@nhk.or.jp \\ Tomoyuki Mishina \\ NHK \\ 1-10-11 Kinuta Setagaya-ku \\ Tokyo, Japan \\ mishina.t-iy@ nhk.or.jp
}

\author{
Kensuke Hisatomi \\ $\mathrm{NHK}$ \\ 1-10-11 Kinuta Setagaya-ku \\ Tokyo, Japan \\ hisatomi.k-ko@nhk.or.jp \\ Yuichi Iwadate \\ $\mathrm{NHK}$ \\ 1-10-11 Kinuta Setagaya-ku \\ Tokyo, Japan \\ iwadate.y-ja@nhk.or.jp
}

\author{
Miwa Katayama \\ NHK \\ 1-10-11 Kinuta Setagaya-ku \\ Tokyo, Japan \\ katayama.m-gm@nhk.or.jp
}

\begin{abstract}
The main purpose of our research was to generate the bullet time of dynamically moving subjects in 3D space or multiple shots of subjects within 3D space. In addition, we wanted to create a practical and generic bullet time system that required less time for advance preparation and generated bullet time in semi-real time after subjects had been captured that enabled sports broadcasting to be replayed. We developed a multi-viewpoint robotic camera system to achieve our purpose. A cameraman controls multiviewpoint robotic cameras to simultaneously focus on subjects in 3D space in our system, and captures multi-viewpoint videos. Bullet time is generated from these videos in semi-real time by correcting directional control errors due to operating errors by the cameraman or mechanical control errors by robotic cameras using directional control of virtual cameras based on projective transformation. The experimental results revealed our system was able to generate bullet time for a dynamically moving player in $3 \mathrm{D}$ space or multiple shots of players within 3D space in volleyball, gymnastics, and basketball in just about a minute. System preparation in calibrating the cameras in advance was finished in just about five minutes. Our system was utilized in the "ISU Grand Prix of Figure Skating 2013/2014, NHK Trophy" live sports program in November 2013. The bullet time of a dynamically moving skater on a large skating rink was generated in semi-real time using our system and broadcast in a replay just after the competition. Thus, we confirmed our bullet time system was more practical and generic.
\end{abstract}

\section{Categories and Subject Descriptors}

I.4.9 [Image Processing and Computer Vision]: Applications

\section{General Terms}

Computer Vision

\section{Keywords}

Bullet time, Multi-view point video, Robotic camera

Permission to make digital or hard copies of all or part of this work for personal or classroom use is granted without fee provided that copies are not made or distributed for profit or commercial advantage and that copies bear this notice and the full citation on the first page. Copyrights for components of this work owned by others than ACM must be honored. Abstracting with credit is permitted. To copy otherwise, or republish, to post on servers or to redistribute to lists, requires prior specific permission and/or a fee. Request permissions from Permissions@acm.org.

CVMP'14, 13-14 November 2014, London, United Kingdom

Copyright 2014 ACM 978-1-4503-3185-2/14/11 ...\$15.00.

http://dx.doi.org/10.1145/2668904.2668932

\section{INTRODUCTION}

Bullet time has been utilized in various media in recent years. Bullet time is a visual effect where the viewpoint moves around frozen or slow motion subjects by capturing them with synchronous multi-viewpoint cameras and switching captured videos along a sequence of cameras. Bullet time is called time slicing or machinegun shooting and has been utilized for sports broadcasting, movies, commercials, and music videos.

The 1999 movie "Matrix" featured actors dodging bullets using bullet time. Multiple cameras were placed around actors circumferentially and multi-viewpoint videos were shot simultaneously. Bullet time was generated by switching the videos along a sequence of cameras. Replay technology companies have developed "freeD"[1], which can capture important scenes in sports clearly in bullet time. For example, assessments of whether a baseball cross play on home base is safe or out can be captured clearly in bullet time. Subjects in "freeD" are captured by multiple cameras and reconstructed into 3D shapes. "freeD" achieves bullet time viewpoints that change smoothly by using the 3D shape data from subjects. Kanade et al. [2][3] developed "Eye Vision" in 2001, which generated the bullet time of dynamically moving American football players. Multiple pan-tilt robotic cameras were controlled in "Eye Vision" to focus on subjects simultaneously and capture multi-viewpoint images of them. Bullet time was generated by switching these multiviewpoint images. Research on free view-point video with a focus on sports has also been addressed in connection with bullet time. Free view-point video allows viewer to interactively watch sports scenes from arbitrary view-points. Ohta et al. [4] and Inamoto and Saito [5][6] implemented a free view-point video system of soccer using a billboard, which was on a single plane and had a live video texture extracted from multiple cameras. The system did not generate complex 3D models to reduce computational cost. However, Grau et al. [7] and Hilton et al. [8] implemented a free view-point video system using $3 \mathrm{D}$ models generated from multiviewpoint videos and achieved high levels of visual quality. Germann et al. [9] interpolated intermediate views from two camera views for rendering novel viewpoints of players in sports matches.

It is difficult for the "Matrix" bullet time system and "freeD" to capture multi-viewpoint images of dynamic moving subjects or multiple shots of subjects within a wide area by using fixed multiple cameras (Figure 1(a)). Although "Eye Vision" solves the 


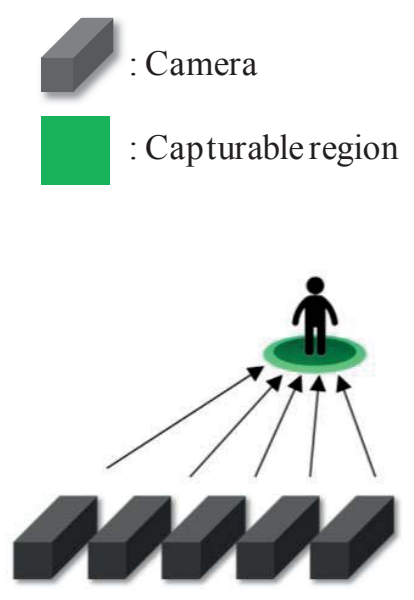

(a)

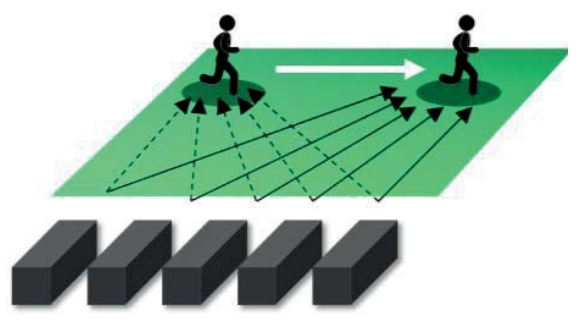

(b)

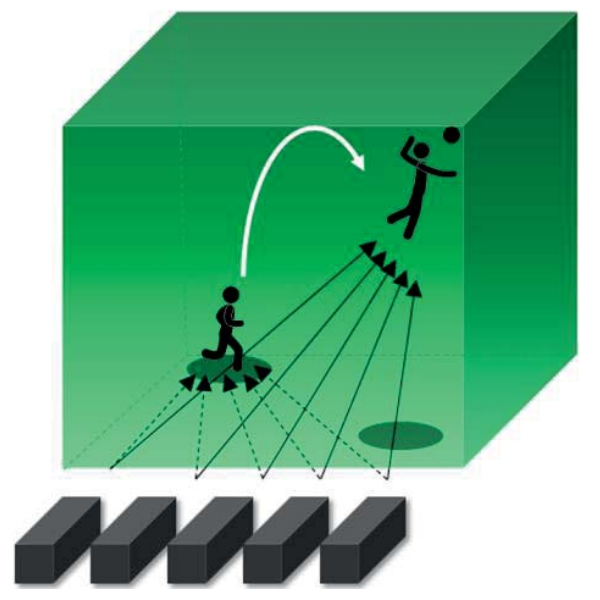

(c)

Figure 1: Capturable region.

(a) Bullet time system of the movie Matrix and freeD. (b) Eye Vision. (c) Our system.

problem by using multiple pan-tilt robotic cameras, it can only capture subjects on a virtual 2D field users have arranged in advance (Figure 1(b)). This is because robotic methods of camera control utilize a restraint condition where subjects are always on the virtual 2D field. Therefore, multiple pan-tilt robotic cameras are placed high above sports stadiums to capture subjects on fields that have been established as virtual 2D fields. Although these cameras can capture subjects on the field by looking down, they cannot capture subjects in 3D space that is located far from the field. "Eye Vision" also has a major problem in that it takes too long to calibrate cameras in advance. When Eye vision was utilized in Japanese baseball broadcasting in 2001, it was reported that camera calibration took four days. Fixed multiple cameras have been utilized in research on free viewpoint video to capture multi-viewpoint images. Therefore, there is a problem where the resolution of subjects is limited because a wide angle is required to capture all content.

The main purpose of our research was to generate the bullet time of dynamically moving subjects in 3D space or multiple shots of subjects within 3D space (Figure 1(c)). In addition, we wanted to create a practical and generic bullet time system that required less time for advance preparation and generated bullet time in semireal time after subjects had been captured that enabled sports broadcasting to be replayed. There were three requirements to achieve our purpose.

1. Control multiple cameras to simultaneously and accurately focus on dynamically moving subjects in $3 \mathrm{D}$ space to generate bullet time viewpoints that change smoothly.

2. Generate the bullet time of playing scenes in processing time where bullet time can be utilized for replays of scenes in live sports broadcasting.

3. Quickly finish advance preparation for camera calibration.

We developed a multi-viewpoint robotic camera system to achieve our purpose. A cameraman controls multi-viewpoint robotic cameras to simultaneously focus on subjects in 3D space in our system, and captures multi-viewpoint videos (Figure 2). Bullet time is generated from these videos in semi-real time by correcting directional control errors due to operating errors by the cameraman or mechanical control errors by robotic cameras using directional control of virtual cameras based on projective transformation. The experimental results revealed our system was able to generate bullet time for a dynamically moving player in $3 \mathrm{D}$ space or multiple shots of players within $3 \mathrm{D}$ space in volleyball, gymnastics, and basketball in just about a minute. System preparation in calibrating the cameras in advance was finished in just about five minutes. Our system was utilized in the live sports program of figure skating. The bullet time of a dynamically moving skater on a large skating rink was generated in semi-real time using our system and broadcast in a replay just after the competition. Thus, we confirmed our bullet time system was more practical and generic than previous bullet time systems.

We present our system in this paper. We first explain the proposed method (Section 2). Then we describe the system (Section 3). The experiment and discussion are presented in Sections 4 and 5. Finally, we summarize the results obtained using our system and briefly touch on future work.
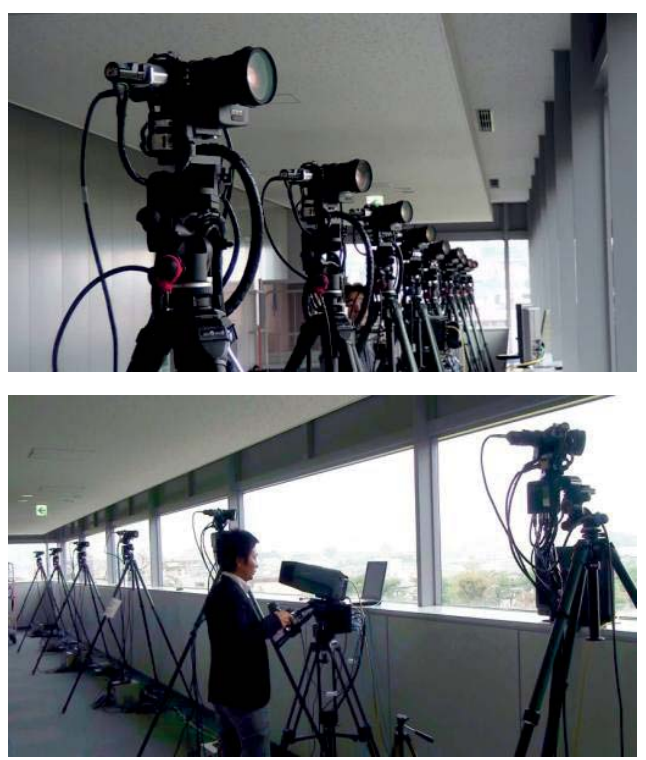

Figure 2 : Multi-viewpoint robotic camera system. 


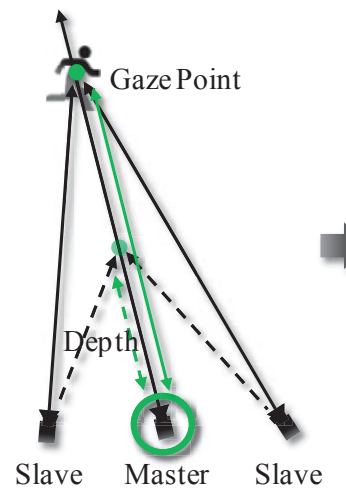

(a)

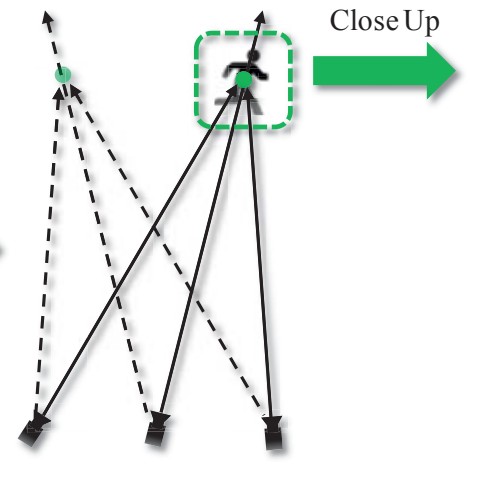

)

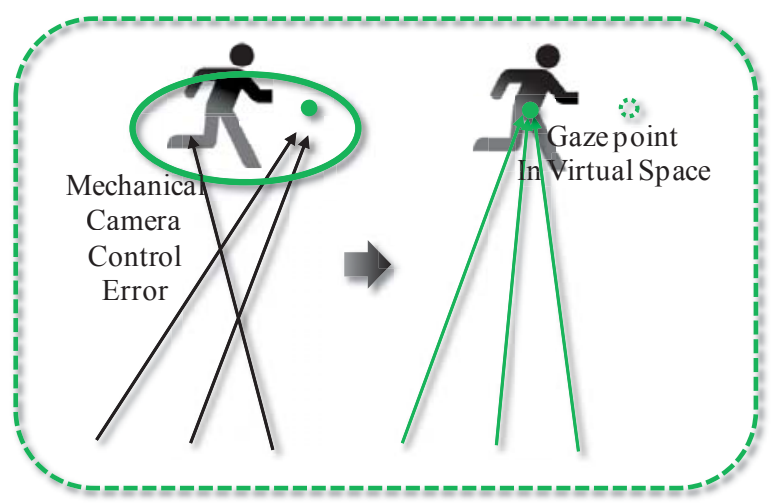

(b)

Figure 3: Camera control.

(a) Directional control of mechanical cameras. (b) Directional control of virtual cameras.

\section{PROPOSED METHOD}

A multi-viewpoint robotic camera system generates bullet time by using both "directional control of mechanical cameras" and "directional control of virtual cameras". Directional control of mechanical cameras captures multi-viewpoint videos by controlling multiple robotic cameras that have pan, tilt and zoom functions to simultaneously focus on subjects in 3D space due to operations by the cameraman (Figure 3(a)). Directional control of virtual cameras corrects directional control errors due to operating errors by the cameraman or mechanically controls errors by robotic cameras using projective transformation (Figure 3(b)).

\subsection{Directional Control of Mechanical Cameras}

\subsubsection{Setting for Robotic Cameras}

The area for capturing subjects is determined by considering where they will be and where they will be moving. Multiviewpoint robotic cameras are placed linearly or in an arc to surround the area of capture. The two optical axes of both end cameras in the setting cross in the center of the capture area at an angular viewpoint that moves around subjects in bullet time. The distance between the cameras and a subject does not need to be the same because the subject size in the image is corrected and equalized by the directional control of virtual cameras, as will be explained later.

\subsubsection{Initial Camera Calibration}

All robotic cameras are defined $\operatorname{Cam}_{n}(1 \leqq n \leqq N)$, where $n$ is the camera number, and $N$ is the number of cameras. All robotic camera directions are manually controlled to capture multiviewpoint images of the same appropriate subject in the area of capture. Camera parameters (rotation matrix $\boldsymbol{R}_{0 n}$ and translation vector $\boldsymbol{t}_{0 n}$ ) and encoder values (pan $P_{0 n}$ and tilt $T_{0 n}$ ) of $C a m_{n}$ are obtained with a method of weak camera calibration[10]. Natural feature points in multi-viewpoint images are utilized for calibration with this method. No calibration objects are needed. Conversion from world coordinate $x$ to camera coordinate $x_{n}$ of $\mathrm{Cam}_{n}$ is defined as:

$$
x_{n}=\left[\begin{array}{ll}
R_{0 n} & t_{0 n}
\end{array}\right]\left[\begin{array}{l}
x \\
1
\end{array}\right](1)
$$

\subsubsection{Cooperative Control of Robotic Cameras}

Figure 3(a) summarizes cooperative control of the robotic cameras. All robotic cameras are cooperatively controlled to constantly follow a gaze point in 3D space. A cameraman can fix the gaze point at an arbitrary position in 3D space by operating the master camera and can capture multi-viewpoint images of subjects by fixing the gaze point on the subject position.

First, master camera $\mathrm{Cam}_{M}$ is selected from cameras, and robotic cameras except for $\mathrm{Cam}_{M}$ are defined as slave cameras. Gaze point $\boldsymbol{g}$ is fixed on optical axis $\boldsymbol{o}_{\boldsymbol{M}}$ of the master camera. $\boldsymbol{o}_{\boldsymbol{M}}$ is calculated from Equations (2), (3), and (4).

$$
\begin{gathered}
\boldsymbol{R}_{\boldsymbol{M}}=\boldsymbol{R}_{\mathbf{0 M}}^{-\mathbf{1}}\left[\begin{array}{ccc}
\cos \left(P_{M}-P_{0 M}\right) & 0 & \sin \left(P_{M}-P_{0 M}\right) \\
0 & 1 & 0 \\
-\sin \left(P_{M}-P_{0 M}\right) & 0 & \cos \left(P_{M}-P_{0 M}\right)
\end{array}\right] \\
{\left[\begin{array}{llc}
1 & 0 & 0 \\
0 & \cos \left(T_{M}-T_{0 M}\right) & -\sin \left(T_{M}-T_{0 M}\right) \\
0 & \sin \left(T_{M}-T_{0 M}\right) & \cos \left(T_{M}-T_{0 M}\right)
\end{array}\right](2)} \\
\boldsymbol{R}_{\boldsymbol{M}}=\left[\begin{array}{lll}
r_{11} & r_{12} & r_{13} \\
r_{21} & r_{22} & r_{23} \\
r_{31} & r_{32} & r_{33}
\end{array}\right] \text { (3) } \boldsymbol{o}_{\boldsymbol{M}}=\left[\begin{array}{l}
r_{13} \\
r_{23} \\
r_{33}
\end{array}\right] \text { (4) }
\end{gathered}
$$

$P_{M}$ and $T_{M}$ are the pan and tilt encoder values of the master camera. $\boldsymbol{g}$ is calculated with Equation (5).

$$
\boldsymbol{g}=-\boldsymbol{R}_{\mathbf{0 M}}^{-1} \boldsymbol{t}_{\mathbf{0 M}}+D \cdot \boldsymbol{o}_{M}(5)
$$

$D$ is the depth value in Equation (5). Depth is the distance between the master camera and the gaze point. The gaze point moves along an optical axis by continuously increasing and decreasing the depth. The cameraman can control the optical direction by panning and tilting the master camera and the gaze point position on the optical axis by changing the depth. The depth is proportionally increased or decreased according to the operating amount of depth in the operating device; it is difficult to intuitively operate the depth. This is because when the gaze point 


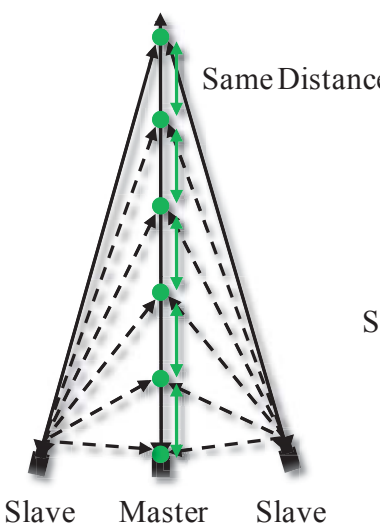

(a)

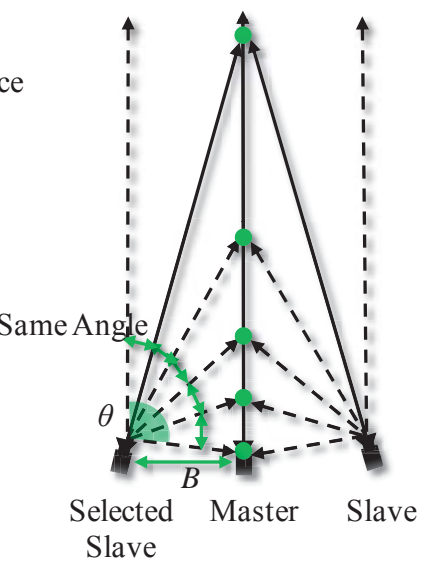

(b)

Figure 4: Depth control.

(a) Proportional increase of depth.

(b) Proportional increase of directional angle of slave cameras.

is near the master camera, the control angle of a slave camera that is following the gaze point changes dynamically, as seen in Figure 4(a). However, when the gaze point is far from the master camera, the control angle of the slave camera hardly changes, as shown in Figure 4(a). As a result, depth is operated according to Equation (6)

$$
D=B \cdot \sin \left(\theta \cdot d / d_{\max }\right) / \sin \left(\theta \cdot\left(1-d / d_{\max }\right)\right)(6)
$$

A slave camera is selected from slave cameras to operate depth according to Equation (6), as Figure 4(b) shows. The $B$ in Equation (6) is the distance between the master camera and the selected slave camera, where $\theta$ is the range of directional control of the selected slave camera, which is the width between the angle when the direction of the selected slave camera is controlled toward the position of the master camera and the angle when the direction of the selected slave camera is controlled to be the same as the optical axis of the master camera. $d$ is the value of depth of the operating device, and $d_{\max }$ is the maximum value of the device. The directional angles of the slave cameras are proportionally increased or decreased according to the operating amount of depth by the operating device by using Equation (6). Therefore, it is possible to intuitively control depth. The directions of all slave camera are controlled to focus on the gaze point. Vector $\boldsymbol{o}_{n}$ toward $\boldsymbol{g}$ from all cameras is calculated with Equation (7).

$$
o_{n}=R_{0 n} \frac{g+R_{0 n}^{-1} t_{0 n}}{\left\|g+R_{0 n}^{-1} t_{0 n}\right\|}(7)
$$

The angles (pan $P_{n}$ and tilt $T_{n}$ ) to control cameras that focus on the gaze point are calculated with Equations (8), (9), and (10).

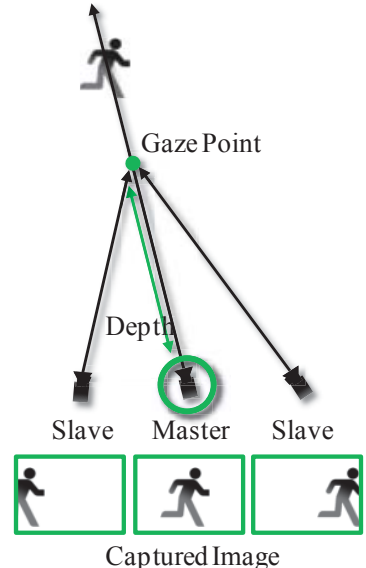

(a)

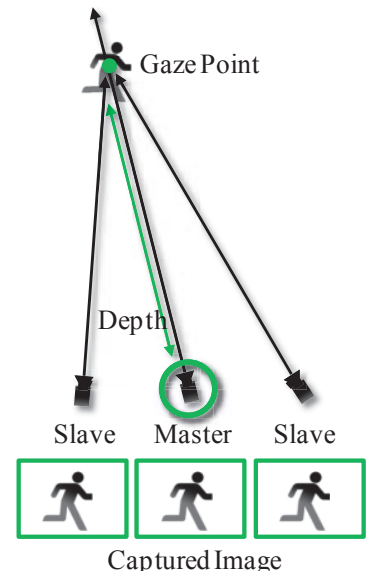

(b)
Figure 5: Depth determination.

(a) When the depth is not correct.

(b) When the depth is correct.

$$
\begin{gathered}
\boldsymbol{o}_{\boldsymbol{n}}=\left[\begin{array}{lll}
e_{n 1} & e_{n 2} & e_{n 3}
\end{array}\right]^{\mathrm{T}}(8) \\
P_{n}=\tan ^{-1}\left(e_{n 1} / e_{n 3}\right)+P_{0 n}(9) \quad T_{n}=\sin ^{-1}\left(e_{n 2}\right)+T_{0 n}(10)
\end{gathered}
$$

All camera directions are controlled by $P_{n}$ and $T_{n}$ to focus on the gaze point.

A camera man captures a subject by panning and tilting the master camera to fix the gaze point on a subject's position and capture multi-viewpoint videos. The gaze point moves along an optical axis to reach the position of the subject according to depth operation. The cameraman operates depth while looking to capture the videos of both the master camera and at least one slave camera. The cameraman can determine whether the depth is correct according to the subject's position in the video captured by the slave camera. For example, when the depth is not correct, the subject is at the center of the video captured by the master camera; however, he/she is not at the center of the video captured by the slave camera (Figure 5(a)). When the depth is correct, on the other hand, the subject is at center in the videos of both the master and slave cameras (Figure 5(b)). The cameraman can determine whether the depth is correct.

\subsection{Directional Control of Virtual Cameras}

\subsubsection{Time and Subject Decisions of Bullet Time}

Captured multi-viewpoint video and focal length at the time of capture are recorded. After recording, a user determines frame $F$ and the subject of bullet time from recorded multi-viewpoint images. The user obtains the image coordinates $\left(u_{a}\right.$ and $\left.v_{a}\right)$ and $\left(u_{b}\right.$ and $v_{b}$ ) of the subject's position from the multi-view point videos of any two cameras, $\mathrm{Cam}_{a}$ and $\mathrm{Cam}_{b}$.

\subsubsection{Camera Calibration}

Camera parameters (rotation matrix $\boldsymbol{R}^{\prime}{ }_{0 n}$, translation vector $\boldsymbol{t}^{\prime}{ }_{\boldsymbol{0} \boldsymbol{n}}$, and focal length $f_{\text {on }}$ ) are obtained with a method of weak camera calibration[10] using the frame $F$ of bullet time and focal length when capture occurred. Conversion from world coordinate $\boldsymbol{x}$ to 
the image coordinates $\left(u_{n}\right.$ and $\left.v_{n}\right)$ of $\mathrm{Cam}_{n}$ is defined in Equations (11), (12), and (13).

$$
\begin{gathered}
\omega\left[\begin{array}{c}
u_{n} \\
v_{n} \\
1
\end{array}\right]=\boldsymbol{S}_{\boldsymbol{n}}\left[\begin{array}{l}
\boldsymbol{x} \\
1
\end{array}\right](11) \\
\boldsymbol{S}_{\boldsymbol{n}}=\boldsymbol{A}_{\mathbf{0} \boldsymbol{n}}^{\prime}\left[\begin{array}{ll}
\boldsymbol{R}_{\mathbf{0} \boldsymbol{n}}^{\prime} & \boldsymbol{t}_{\mathbf{0} \boldsymbol{n}}^{\prime}
\end{array}\right](12) \\
\boldsymbol{A}_{\mathbf{0} \boldsymbol{n}}^{\prime}=\left[\begin{array}{ccc}
f_{0 n} & 0 & C_{x} \\
0 & f_{0 n} & C_{y} \\
0 & 0 & 1
\end{array}\right](13)
\end{gathered}
$$

Here, $\omega$ is the image distance, $f_{0 n}$ is the focal length of $\mathrm{Cam}_{n}$, and $\left(C_{x}, C_{y}\right)$ is the image center.

\subsubsection{Projective Transformation}

A gaze point is re-set on the subject position selected in Subsection 2.2.1. All camera directions are re-controlled virtually to focus on $\boldsymbol{g}^{\prime}$ using projective transformation. $\boldsymbol{g}^{\prime}$ is calculated with Equations (14), (15), and (16).

$$
\boldsymbol{g}^{\prime}=\boldsymbol{M}^{+} \boldsymbol{b}(14)
$$

$$
\boldsymbol{M}=\left[\begin{array}{lll}
S_{a 31} u_{a}-S_{a 11} & S_{a 32} u_{a}-S_{a 12} & S_{a 33} u_{a}-S_{a 13} \\
S_{a 31} v_{a}-S_{a 21} & S_{a 32} v_{a}-S_{a 22} & S_{a 33} v_{a}-S_{a 23} \\
S_{b 31} u_{b}-S_{b 11} & S_{b 32} u_{b}-S_{b 12} & S_{b 33} u_{b}-S_{b 13} \\
S_{b 31} v_{b}-S_{b 21} & S_{b 32} v_{b}-S_{b 22} & S_{b 33} v_{b}-S_{b 23}
\end{array}\right]
$$

$$
\boldsymbol{b}=\left[\begin{array}{l}
S_{a 14}-S_{a 34} u_{a} \\
S_{a 24}-S_{a 34} v_{a} \\
S_{b 14}-S_{b 34} u_{b} \\
S_{b 24}-S_{b 34} v_{b}
\end{array}\right]
$$

$S_{n i j}$ represents the $i$ row and $j$ column elements of $\boldsymbol{S}_{\boldsymbol{n}}$, and $\boldsymbol{M}^{+}$is the generalized inverse of $\boldsymbol{M}$. When the camera direction is controlled virtually, the vertical axis to the ground is calculated to keep the camera horizontal to the ground. The vertical axis is calculated by using the outer product of the tilt axis of any two cameras, $\mathrm{Cam}_{s}$ and $\mathrm{Cam}_{t}$, because the tilt axis of a pan/tilt robotic camera is horizontal to the ground. The vertical axis is calculated with Equations (17) and (18).

$$
\boldsymbol{R}_{0 n}^{\prime}=\left[\begin{array}{l}
r_{n 1} \\
r_{n 2} \\
r_{n 3}
\end{array}\right](17) \quad v=\frac{r_{s 1} \times r_{t 1}}{\left\|r_{s 1} \times r_{t 1}\right\|}(18)
$$

The rotation matrix when the camera direction is controlled to virtually focus on $\boldsymbol{g}^{\prime}$ is calculated with Equations (19), (20), (21), and (22).

$$
\boldsymbol{R}_{\boldsymbol{n}}^{\prime}=\left[\begin{array}{c}
\boldsymbol{e}_{\boldsymbol{n} x}{ }^{\mathrm{T}} \\
\boldsymbol{e}_{\boldsymbol{n} \boldsymbol{y}}^{\mathrm{T}} \\
\boldsymbol{e}_{\boldsymbol{n} \boldsymbol{z}}^{\mathrm{T}}
\end{array}\right]
$$

$$
e_{n z}=\frac{g^{\prime}+R_{0 n}^{\prime}-1 t_{0 n}^{\prime}}{\left\|g^{\prime}+R_{0 n}^{\prime-1} t_{0 n}^{\prime}\right\|} \text { (20) } e_{n x}=\frac{e_{n z} \times v}{\left\|e_{n z} \times v\right\|} \text { (21) } e_{n y}=\frac{e_{n z} \times e_{n x}}{\left\|e_{n z} \times e_{n x}\right\|}
$$

The $\boldsymbol{e}_{n x}, \boldsymbol{e}_{n y}$, and $\boldsymbol{e}_{n z}$ are the tilt, pan, and roll axis of each camera. When the camera direction is virtually controlled to focus on $\boldsymbol{g}^{\prime}$, the subject size of all cameras is equalized to the subject size of the master camera, $\mathrm{Cam}_{M} . A_{n}^{\prime}$ is calculated by rectifying each camera's focal length according to the distance between the camera and gaze point by using Equations (23) and (24).

$$
f_{n}=k \cdot f_{0 M} \cdot \frac{\| \boldsymbol{g}^{\prime}+\boldsymbol{R}_{0 n}^{\prime}-1}{\left\|\boldsymbol{t}_{0 n}^{\prime}\right\|} \quad(23) \quad \boldsymbol{A}_{\boldsymbol{n}}^{\prime}=\left[\begin{array}{ccc}
f_{n} & 0 & C_{x} \\
0 & f_{n} & C_{y} \\
0 & 0 & 1
\end{array}\right](24)
$$

Here, $k$ is the zoom rate after projective transformation. Projective transformation is carried out on the videos of all cameras to virtually control the camera direction toward $\boldsymbol{g}^{\prime}$ by using Equations (25) and (26). The $u_{n}$ and $v_{n}$ are the image coordinates of a pixel before projective transformation, $u_{n}^{\prime}$ and $v_{n}^{\prime}$ are the image coordinates of a pixel after projective transformation.

$$
H_{n}=A_{n}^{\prime} R_{n}^{\prime} R_{0 n}^{\prime-1} A_{0 n}^{\prime-1}(25)
$$

$$
\left[\begin{array}{c}
u^{\prime}{ }_{n} \\
v_{n}^{\prime} \\
1
\end{array}\right]=\boldsymbol{H}_{n}\left[\begin{array}{c}
u_{n} \\
v_{n} \\
1
\end{array}\right]
$$

\subsubsection{Bullet Time Video Generation}

Bullet time video is generated by editing projective transformation images. Figure 6 summarizes the editing method. Bullet time video is generated by replaying projective transformation images along the camera pass in Figure 6. All images on the camera pass are projectively transformed and images from Cam1 are replayed. When the replay frame reaches bullet time frame $F$ selected in Subsection 2.2.1, images are replayed from Cam1 to CamN. Finally, images from $C a m N$ are replayed.

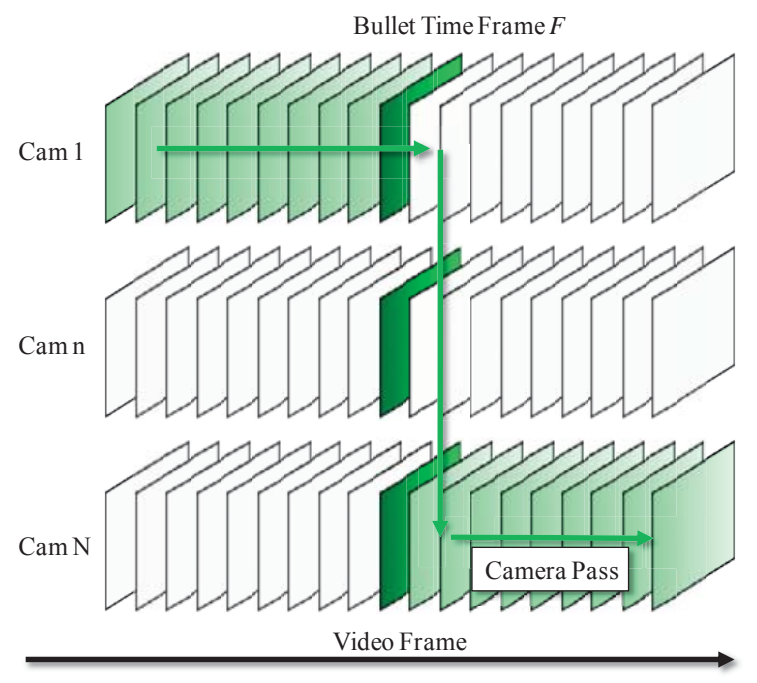

Figure 6: Editing method of bullet time. 


\section{SYSTEM}

A multi-viewpoint robotic camera system mainly consists of multi-viewpoint robotic cameras (Figure 7), an operation interface (Figure 8), and a bullet time processor (Figure 9). Multi-viewpoint robotic cameras are multiple robotic cameras that have small high-definition (HD) cameras mounted on an electric dynamic pan-tilt platform and have a board computer. The pan, tilt, zoom, focus and iris can be remotely controlled by external signals. A servo motor was introduced to accurately control the camera direction. Nine robotic cameras have recently been equipped. The operation interface can control the pan, tilt, depth of the master camera and the zoom of all cameras and is an operation interface for virtual reality modified for our system. Depth is operated by focus demand. Focus is automatically controlled by depth. Therefore, multi-viewpoint robotic cameras can be operated by cameramen in almost the same way as ordinary TV cameras. The bullet time processor device is a workstation that generates bullet time video by controlling the direction of virtual cameras to capture videos obtained from recorders. The bullet time processor device has software that encourages quick user operation (time and subject decisions in bullet time) and a GPU for high speed projective transformation to generate bullet time videos in semireal time. The software can freely change the direction of viewpoints in bullet time, change the replay speed, and change the digital zoom rate at any time for any reason.

Figure 10 is a diagram of the system. The operation interface transmits pan, tilt, zoom, and depth data to the master camera. The master camera achieves control based on received data and transmits pan, tilt, zoom, and depth data to a personal computer (PC) that calculates the gaze point. The PC calculate the gaze point and transmits the world coordinates of the gaze point calculated from the pan, tilt, and depth to the slave cameras. The direction of the slave cameras is controlled based on the received data. Videos captured by multi-viewpoint robotic cameras and the focal length at the time of capture are recorded by a recorder. The bullet time processor consists of a user interface, a weak cameracalibration component, a projective-transformation component, and a bullet time video-generation component. The frames of bullet time and the subject are determined from the user interface.
The frame data are transmitted to the weak camera-calibration and projective transformation components. The image coordinates of the subject's position are transmitted to the projective transformation component. The weak camera- calibration component obtains both multi-view point images and the focal length of the received frame number and transmits the camera parameters calculated by weak camera calibration to the projective transformation component. The projective transformation component obtains multi-viewpoint images of the received frame number from the recorder and conducts projective transformation using the received camera parameters. The bullet time video generation component generates bullet time videos by editing the projective transformation images.

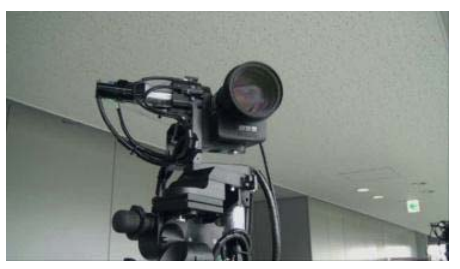

Figure 7: Robotic camera.

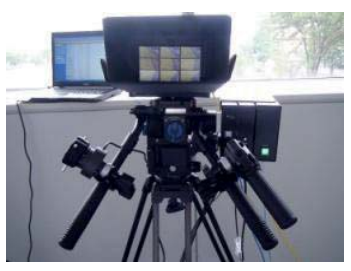

Figure 8: Operating Interface.

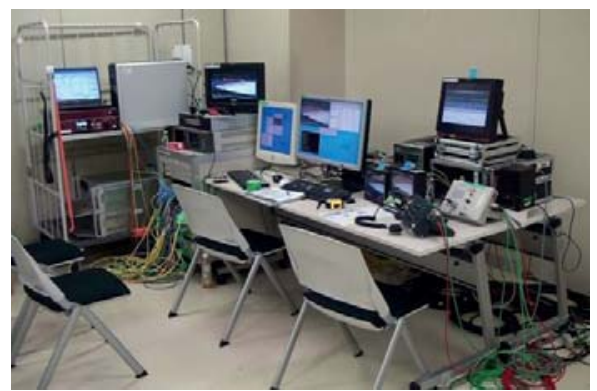

Figure 9: Bullet time processor.

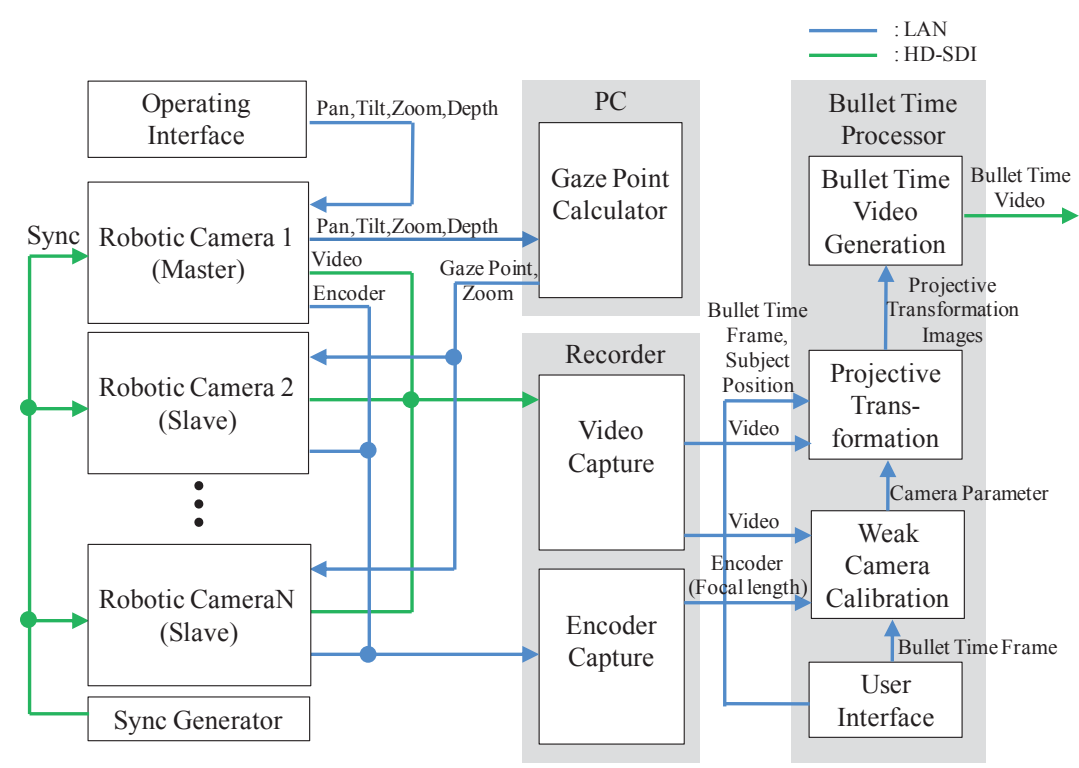

Figure 10 : Diagram of the system. 


\section{EXPERIMENT}

\subsection{Bullet Time Generation of Sports Scene}

We captured a dynamically moving player in 3D space and multiple shots of players within 3D space in a volleyball game and generated bullet time videos to evaluate the performance of our system. Nine robotic cameras were placed in a 90 degree arc, as shown in Figure 11. The distance between cameras and subjects was about $11 \mathrm{~m}$, and the distance between neighboring cameras was $2.3 \mathrm{~m}$. Figure 12 shows the generated bullet time videos. We also performed an experiment under similar

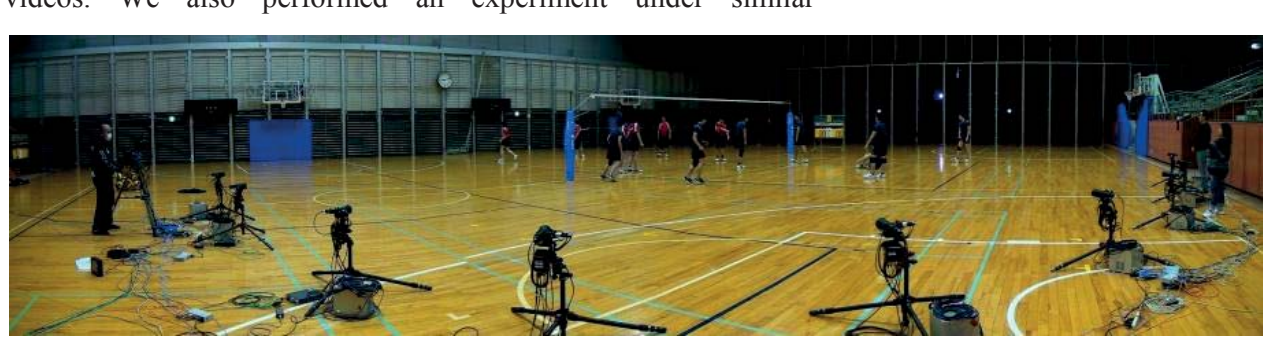

Figure 11 : Camera position. conditions using subjects doing gymnastics and playing basketball and generated bullet time videos. The bullet time videos are shown in Figure 13, 14. The experimental results revealed that our system could generate the bullet time of a dynamically moving player in 3D space or multiple shots of players within 3D space from a volleyball game in just about a minute. System preparation in calibrating the cameras in advance was finished in just about five minutes. We were also similarly able to generate the bullet time of subjects doing gymnastics and playing basketball.
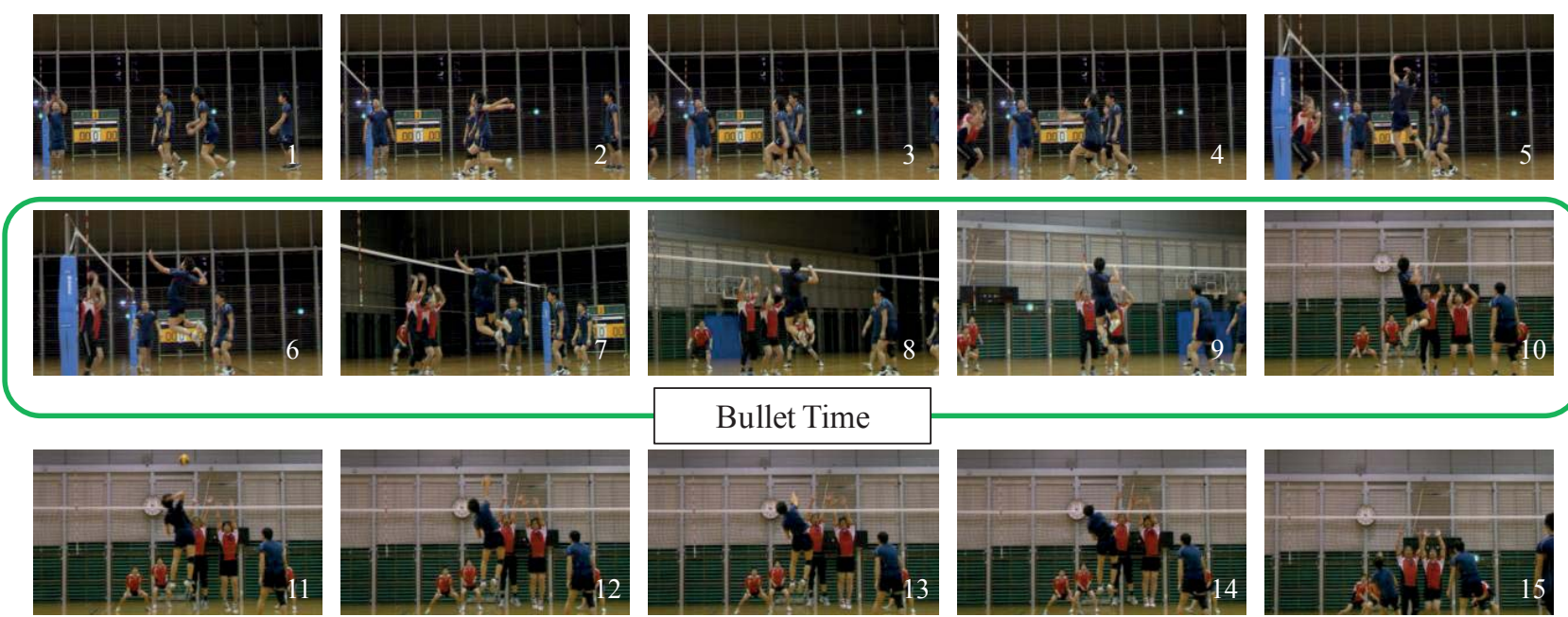

Figure 12: Bullet time of volley ball scene. (The number is frame flow)
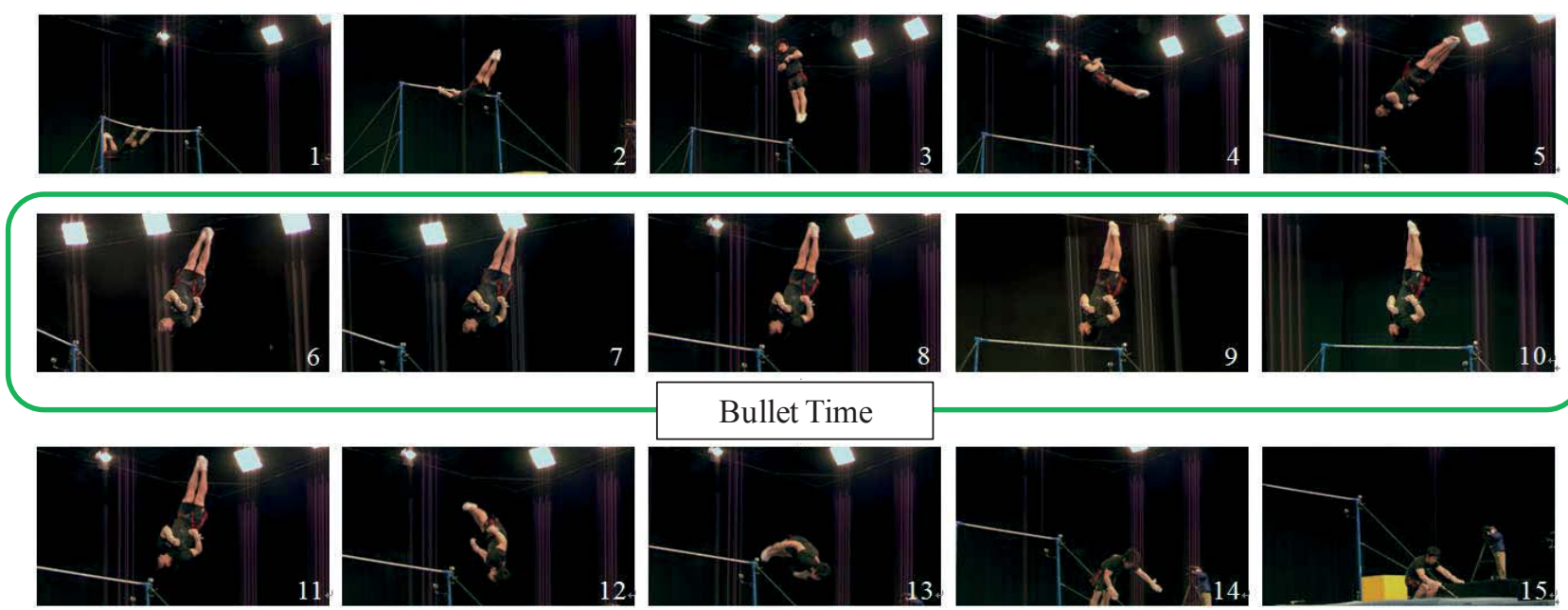

Figure 13: Bullet time of gymnastics. 

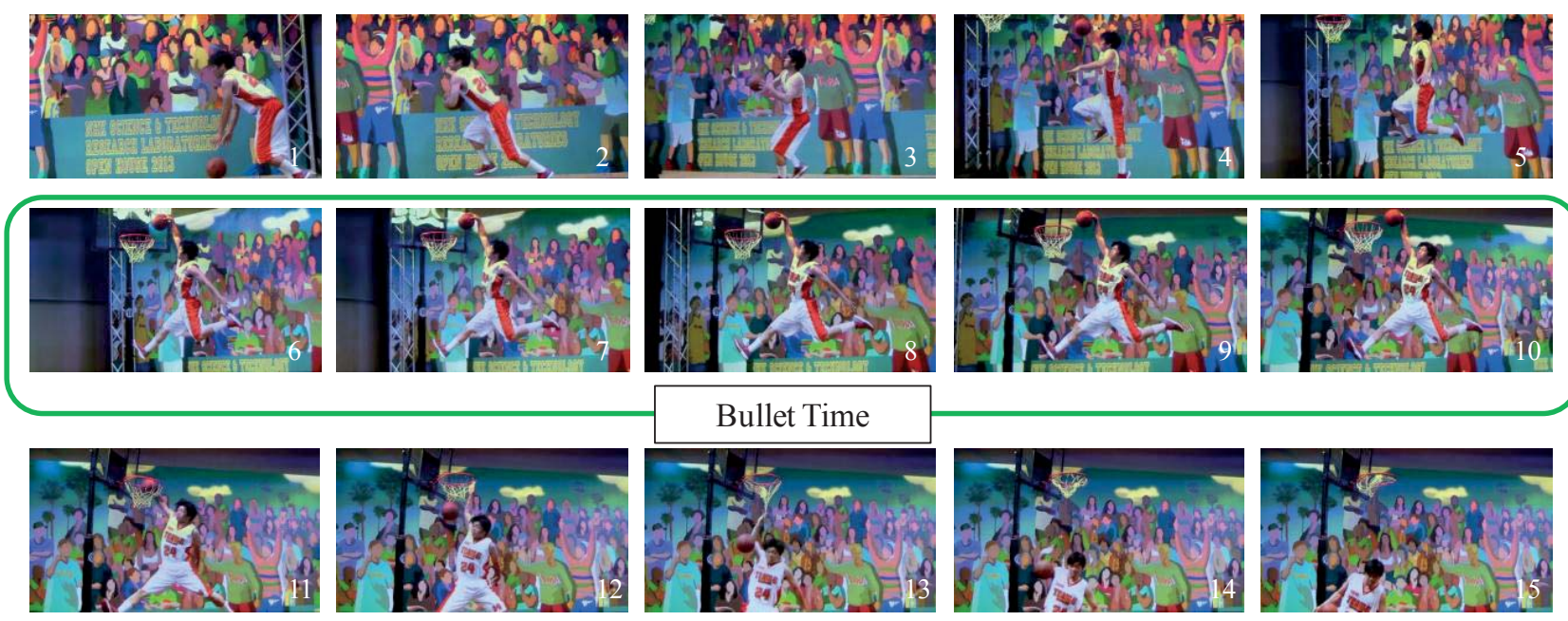

Figure 14: Bullet time of basket ball.

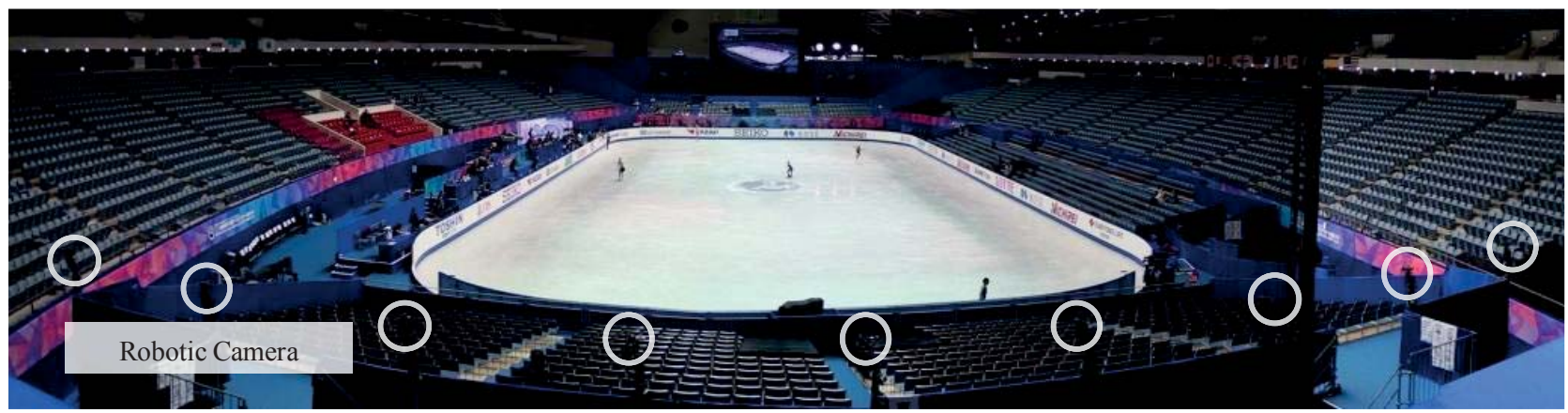

Figure 15: Camera position.
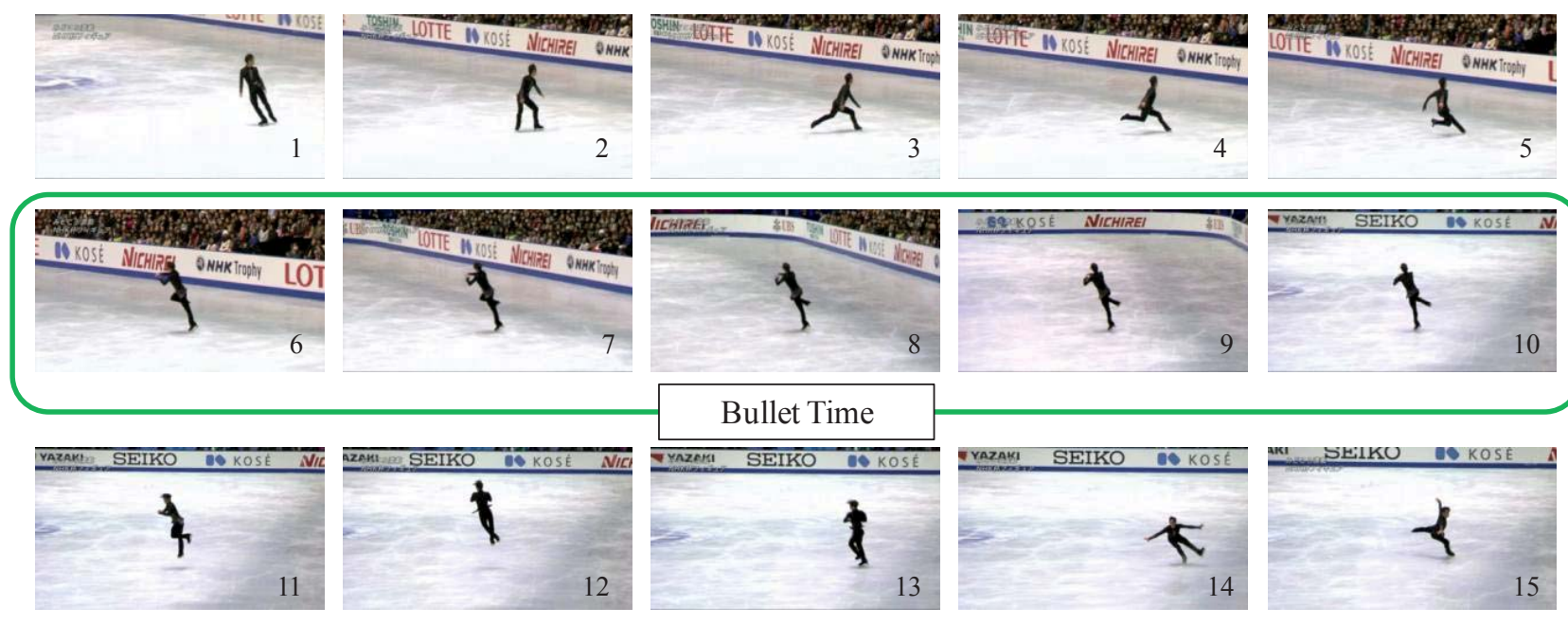

Figure 16: Bullet time of figure skating. (The number is frame flow)

\subsection{Utilize System for Broadcasting}

Our system was utilized in the "ISU Grand Prix of Figure Skating 2013/2014, NHK Trophy" live sports program in November 2013. Nine robotic cameras were placed on the seats of sports fans in a line, as shown in Figure 15. The distance between cameras and subjects was about $30 \mathrm{~m}$, and the distance between neighboring cameras was $2 \mathrm{~m}$. The bullet time of a dynamically moving skater on a large skating rink was generated in semi-real time using our system and broadcast in a replay just after the competition, as shown in Figure 16. The take-off pose, aerial pose, and landing pose in a skate jump were clearly captured for viewers in bullet time. 


\section{DISCUSSION}

The main purpose of our research was to generate the bullet time of dynamically moving subjects in 3D space or multiple shots of subjects within $3 \mathrm{D}$ space. In addition, we wanted to create a practical and generic bullet time system that required less time for advance preparation and generated bullet time in semi-real time after subjects had been captured that enabled sports broadcasting to be replayed. There were three requirements to achieve our purpose aforementioned.

We evaluated whether our system could satisfy the first requirement cited in Section 1. Requirement 1 was to simultaneously and accurately control multiple cameras that focused on dynamically moving subjects in 3D space to generate bullet time viewpoints that changed smoothly. Our system made it possible to accurately control the directions of multi-viewpoint cameras to focus on dynamically moving subjects in 3D space and capture multiple shots of them within 3D space in volleyball, gymnastics, basketball, and figure skating by both mechanically controlling the camera direction and virtually controlling the camera direction of multi-viewpoint robotic cameras. We measured the Euclidian distance between a gaze point and the center of the images in a bullet time scene to evaluate the accuracy with which the camera direction could be controlled, as shown in Figure 12. The average Euclidian distance of nine camera images was 1.4 pixels. This error in the control of the camera direction did not affect the generation of bullet time. Our system made it possible to accurately control the camera direction. Our system satisfied Requirement 1. Requirement 2 was to generate the bullet time of a playing scene in processing time where the bullet time could be utilized to replay the scene during live sports broadcasting. Our system made it possible to generate bullet time in just about a minute after capture had finished. The bullet time was utilized for replay just after competition had finished in the live sports program of figure skating. Our system satisfied requirement 2 . Requirement 3 was to finish preparation of camera calibration in advance in a short time. Our system made it possible to finish system preparation to calibrate cameras in advance in just about five minutes. Our system satisfied Requirement 3. We confirmed that our system satisfied all three requirements.

We compared the technology of our system with previous technologies. The bullet time systems of the movie "Matrix" and "freeD" made it difficult to capture dynamically moving subjects in large spaces or capture multiple shots of subjects within large spaces. Although "Eye Vision" solved the problem, it could only capture subjects on a virtual 2D field user arranged in advance. Our system, on the other hand, made it possible to capture dynamically moving subjects in $3 \mathrm{D}$ space or capture multiple shots of players within 3D space and it solved the problems with previous technologies. Therefore, our system was able to achieve the bullet time of aerially dynamic moving players in volleyball and gymnastics by following them with pan and zoom shots. "Eye Vision" also has the problem in that it takes too long to calibrate cameras during advance preparation. When "Eye Vision" was utilized in Japanese baseball broadcasting in 2001, it was reported that camera calibration took four days. However, our system made it possible to finish system preparation in calibrating cameras in advance in just about five minutes and it solved the problem "Eye Vision" has. Thus, we confirmed our bullet time system was more practical and generic than previous bullet time systems. Fixed multiple cameras were utilized to capture multi-viewpoint images in the research on free viewpoint video. Therefore, there is a problem where the resolution of subjects is limited because a wide angle is required to capture all content. Our system is able to solve this problem by allowing operators to capture the region of interest with an appropriate viewing angle using multi-view point robotic cameras.

\section{CONCLUSION}

We developed a multi-viewpoint robotic camera system to achieve our purpose. A cameraman controls multi-viewpoint robotic cameras to simultaneously focus on subjects in 3D space in our system, and captures multi-viewpoint videos. Bullet time is generated from these videos in semi-real time by correcting directional control errors due to operating errors by the cameraman or mechanical control errors by robotic cameras using directional control of virtual cameras based on projective transformation. The experimental results revealed our system was able to generate bullet time for a dynamically moving player in 3D space or multiple shots of players within 3D space in volleyball, gymnastics, and basketball in just about a minute. System preparation in calibrating the cameras in advance was finished in just about five minutes. Our system was utilized in the live sports program of figure skating. The bullet time of a dynamically moving skater on a large skating rink was generated in semi-real time using our system and broadcast in a replay just after the competition. As a result, we confirmed our system was able to achieve the bullet time of dynamically moving subjects in 3D space or multiple shots of players within 3D space. In addition, our system took a short time in advance preparation and generated bullet time in semi-real time after capture had been completed. Thus, we confirmed our bullet time system was more practical and generic than previous bullet time systems.

We intend to develop a system that can generate higher quality bullet time in the future. We therefore intend to introduce $8 \mathrm{~K}$, which have higher resolution and higher frame rates than high vision cameras, to multi-viewpoint robotic camera systems. We also intend to generate bullet time in which viewpoints are changed smoothly by generating interpolation videos between the viewpoints of multi-viewpoint cameras. We aim to utilize the bullet time system for the Tokyo Olympics in 2020.

\section{REFERENCES}

[1] http://replay-technologies.com/

[2] T. Kanade, P. Rander, and P. Narayanan. Virtualized reality: Constructing virtual worlds from real scenes. IEEE Multimedia, 4(1):34-47, 1997.

[3] R.Collins and T.Kanade. Multi-Camera Tracking and Visualization for Surveillance and Sports. Fourth International Workshop on Cooperative Distributed Vision Proceedings, page 27-55, March 2001.

[4] Y. Ohta, I. Kitahara, Y. Kameda, H. Ishikawa and T. Koyama. Live 3D Video in Soccer Stadium. International Journal of Computer Vision (IJCV), 75(1):173-187, 2007

[5] K. Kimura and H. Saito. Player viewpoint video synthesis using multiple cameras. in IEEEur. Conf. Vis. Media Prod., page 112-121, 2005.

[6] N. Inamoto and H. Saito. Virtual viewpoint replay for a soccer match by view interpolation from multiple cameras. IEEE Trans. Multimedia, 9(6):1155-1166, 2007.

[7] O. Grau, M. Prior-Jones, and G. Thomas. 3d modelling and rendering of studio and sport scenes for tv applications. in Proc. of WIAMIS. 
[8] A. Hilton, J. Guillemaut, J. Kilner, O. Grau, and G. Thomas. 3D-TV production from conventional cameras for sports broadcast. IEEE Trans. Broadcasting, 57(2):462-476, Jun 2011.

[9] M. Germann, A. Hornung, R. Keiser, R. Siegler, S. Wurmlin, and M. Gross. Articulated billboards for video-based rendering. Comput.Graphics Forum, 29(2):585-594, 2010.

[10] Noah Snavely, Steven M. Seitz and Richard Szeliski. Photo Tourism: Exploring image collections in 3D. ACM Transactions on Graphics (Proceedings of SIGGRAPH 2006), 25(3), 2006.

[11] K. Tomiyama, K. Hisatomi, M. Katayama, Y. Iwadate, T. Hayashida, H. Maruyama, T. Goji and T. Yoshida. Advanced video image technologies for sports TV. NAB Show Broadcast Engineering Conference (BEC) Proceedings, page 193-198, 2008. 